\title{
Topological properties of face-centred cubic lattice
}

\author{
Muhammad Kamran Siddiqui*1 (D), Muhammad Imran²,3 (D), Muhammad Saeed ${ }^{4}$ (C) \\ ${ }^{1}$ Department of Mathematics, COMSATS University Islamabad, Lahore Campus, 54000, Pakistan \\ ${ }^{2}$ Department of Mathematical Sciences, United Arab Emirates University, P. O. Box 15551, Al Ain, \\ United Arab Emirates \\ ${ }^{3}$ Department of Mathematics, School of Natural Sciences (SNS), National University of Sciences and \\ Technology (NUST), Sector H-12, Islamabad, 46000, Pakistan \\ ${ }^{4}$ Department of Basic Sciences, Riphah International University Islamabad, 46000 Pakistan
}

\begin{abstract}
Face-centred cubic lattice $F C C(n)$ has attracted large attention in recent years owing to its distinguished properties and non-toxic nature, low-cost, abundance, and simple fabrication process. The graphs of face-centred cubic lattice contain cube points and face centres. A topological index of a chemical graph $G$ is a numeric quantity related to $G$ which describes its topological properties. In this paper, using graph theory tools, we determine the topological indices namely, Randic index, atomic bond connectivity index, Zagreb types indices, Sanskruti index for face-centred cubic lattice $F C C(n)$.
\end{abstract}

Mathematics Subject Classification (2010). 05C12, 05C90

Keywords. Randic index, atomic bond connectivity index, Zagreb types indices, Sanskruti index, face-centred cubic lattice $F C C(n)$.

\section{Introduction}

Mathematical chemistry is a branch of theoretical chemistry in which we discuss and predict the chemical structure by using mathematical tools. Chemical graph theory is a branch of mathematical chemistry in which we apply tools of graph theory to model the chemical phenomenon mathematically. This theory contributes a prominent role in the fields of chemical sciences.

By their help some physical properties, e.g., boiling point, can be predicted based on the structure of the molecules. Mathematical and computational methods are successfully used to model and predict the structure of matter in atomic level [5]. The structures of molecules, from mathematical point of view, are graphs. Graph theory is used in almost every field of science and it is also heavily used in practice, both for simulations and engineering solutions. A graph in this context is made up of vertices or nodes and lines called edges that connect them. Digital geometry deals with regular tessellations, i.e., graphs with regular, periodic structures, and in this way, it is closely related to crystallography.

\footnotetext{
*Corresponding Author.

Email addresses: kamransiddiqui75@gmail.com (M.K. Siddiqui), imrandhab@gmail.com (M. Imran), msaeed42091@gmail.com (M. Saeed)

Received: 22.04.2018; Accepted: 18.10.2018
} 
Each structural formulas that incorporate covalent bonded compounds or atoms are diagrams. Thusly they are called molecular graphs or, basic diagrams or its better to state constitutional graphs. In chemistry, graph theory gives the premise to definition, numeration, systematization of the issue close by, it gives the way toward organizing laws or standards as per a framework or arranging, terminology, it gives the association between the compounds or atoms, and PC programming. The significance graph theory for science stands fundamentally from the presence of isomerism, which is supported by chemical graph theory.

A graph $G(V, E)$ with vertex set $V$ and edge set $E$ is connected, if there exists a connection between any pair of vertices in $G$. A network is simply a connected graph having no multiple edges and no loops. For a graph $G$, the degree of a vertex $v$ is the number of edges incident to $v$ and denoted by $\operatorname{deg}(v)$. A molecular graph is hydrogen depleted chemical structure in which vertices denote atoms and edges denote the bonds.

A molecular graph is a set of vertices representing the atoms in a molecule and a set of edges representing the covalent bonds between the atoms. Not only molecules can be represented by graphs, there are some elements that form lattice structures in their crystals. Carbon (in diamond) and Silicon have cubic lattice structure known as the diamond structure.

A topological index is a numeric quantity associated with a graph which characterize the topology of graph and is invariant under graph automorphism. A topological index $\operatorname{Top}(G)$ of a graph $G$, is a number with the property that for every graph $H$ isomorphic to $G$, $\operatorname{Top}(H)=\operatorname{Top}(G)$. The concept of topological index came from work done by Wiener [31] while he was working on boiling point of paraffin. He named this index as path number. Later on, the path number was renamed as Wiener index. The Wiener index is the first and most studied topological index, both from theoretical point of view and applications, and defined as the sum of distances between all pairs of vertices in $G$, see for details [8].

The first and oldest degree based index is introduced by Milan Randic [23] in 1975 and is defined in the following equation.

$$
R_{-\frac{1}{2}}(G)=\sum_{s t \in E(G)} \frac{1}{\sqrt{d(s) \times d(t)}} .
$$

In 1988, Bollobás et al. [4] and Amic et al. [1] proposed the general Randić index independently. For more details about Randić index, its properties and important results $[6,21]$. The general Randić index is defined as:

$$
R_{\alpha}(G)=\sum_{s t \in E(G)}(d(s) \times d(t))^{\alpha} .
$$

Where $\alpha=1,-1, \frac{1}{2}, \frac{-1}{2}$.

The atom bond connectivity index is of vital importance and introduced by Estrada et al. [10]. It is defined as:

$$
A B C(G)=\sum_{s t \in E(G)} \sqrt{\frac{d(s)+d(t)-2}{d(s) \times d(t)}} .
$$

The geometric arithmetic index $G A$ of a graph $G$ is introduced by Vukičević et al. [28]. It is defined as:

$$
G A(G)=\sum_{s t \in E(G)} \frac{2 \sqrt{d(s) d(t)}}{d(s)+d(t)} .
$$

One of the oldest topological index is the first zagreb index an introduced by I. Gutman and N. Trinajstic on based degree of vertices of $G$ in 1972. The Zagreb indices were 
conceived in 1972 by Gutman and Trinajestic in [14,17], they characterized as:

$$
\begin{aligned}
& M_{1}(G)=\sum_{s t \in E(G)}(d(s)+d(t)) . \\
& M_{2}(G)=\sum_{s t \in E(G)}(d(s) \times d(t)) .
\end{aligned}
$$

For more details see $[15,16]$.

In 2008, Došlić put forward the first Zagreb coindex and second Zagreb coindex, defined as [9]:

$$
\begin{aligned}
& \overline{M_{1}}=\overline{M_{1}}(G)=\sum_{s t \notin E(G)}[d(s)+d(t)] . \\
& \overline{M_{2}}=\overline{M_{2}}(G)=\sum_{s t \notin E(G)}(d(s) \cdot d(t)) .
\end{aligned}
$$

In 2016, I. Gutman et al, [16] proves the following Theorems:

Theorem $1.1([16])$. Let $G$ be a graph with $|V(G)|$ vertices and $|E(G)|$ edges. Then

$$
\overline{M_{1}}(G)=2|E(G)|(\mid V(G)-1)-M_{1}(G) .
$$

Theorem $1.2([16])$. Let $G$ be a graph with $|V(G)|$ vertices and $|E(G)|$ edges. Then

$$
\overline{M_{2}}(G)=2|E(G)|^{2}-\frac{1}{2} M_{1}(G)-M_{2}(G) .
$$

In 2016, S. M. Hosamani [18] introduced the Sanskruti index $S(G)$ for a molecular graph $G$, defined as:

$$
S(G)=\sum_{s t \in E(G)}\left(\frac{S_{s} \times S_{t}}{S_{s}+S_{t}-2}\right)^{3} .
$$

where $S_{s}=\sum_{s t \in E(G)} d(t)$ and $S_{t}=\sum_{s t \in E(G)} d(s)$.

Nowadays there is an extensive research activity on above mention topological indices, see also $[2,3,11-13,19,20,24-27,29,30]$.

\section{Main results}

In this section, additive topological indices mainly Randic index, atomic bond connectivity index, Zagreb types indices and Sanskruti index are computed. Moreover, close formulas are derived which are helpful for the analysis of topological properties of molecular structures of $F C C(n)$.

\subsection{Face-centred cubic lattice}

Face-centred cubic lattice $F C C(n)$ consists of unit cells that are cubes with an atom at each corner of the cube and an atom in the centre of each face of the cube, see Figure 1. In our graphs vertices (points) represent the atoms; the terms, cube vertices (cube points) and face centres (or face centre points) will be used, respectively. Edges connect the closest (neighbour) atoms. In fact, $F C C(n)$ structure has the largest packing density in the three dimensional space: this is one of the most efficient structures to pack same size spheres in a volume [7,22], as it can be seen in Figure 1. Therefore, this structure is also known as cubic closest-packed crystal structure. Metals with $F C C(n)$ structure include: Aluminium, Copper, Gold, Nickel and Silver. In this paper, we are using graphs that represent rows of unit cells of the $F C C(n)$ lattice (i.e., the dimension of our space is $(n \times 1 \times 1)$ unit cells). The molecular graph of face-centred cubic lattice $F C C(n)$ has total 
$9 n+5$ atoms(vertices), among them, the number of vertices of degree 4 are $5 n+1$, the number of vertices of degree 6 are 8 and the number of vertices of degree 9 are $4 n-4$. Also on the other hand total number of bodes(edges) are $28 n+8$, see Figure 2 . To find the

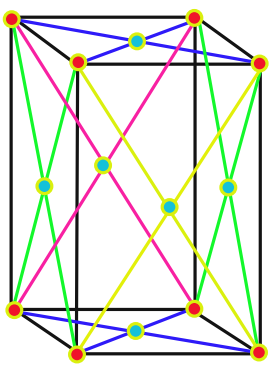

(a)

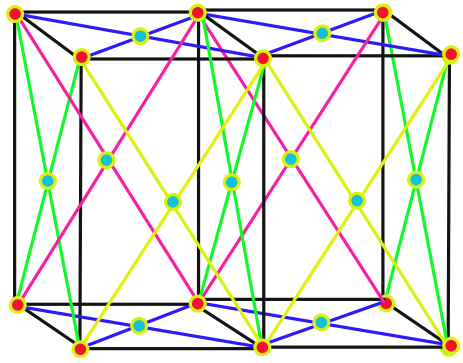

(b)

Figure 1. (a) Unit cell of $F C C(n) \quad$ (b) Face-centred cubic lattice $F C C(2)$.
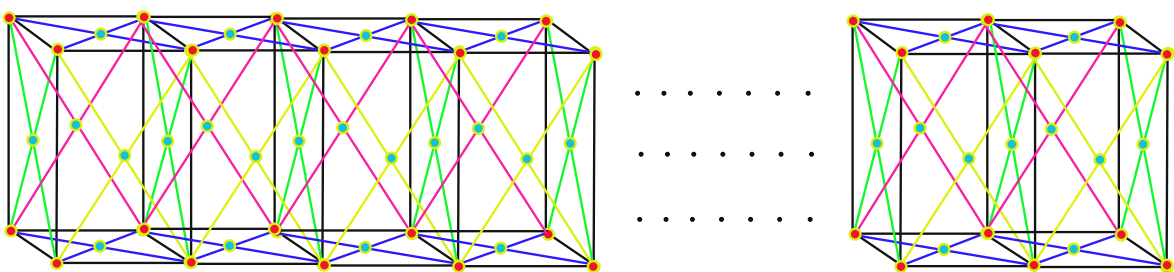

Figure 2. Face-centred cubic lattice $F C C(n)$.

abstracted indices we will partition the edges of $F C C(n)$ The first edge partition contains 24 edge $s t$, where $\operatorname{deg}(s)=4$ and $d(t)=6$. The second edge partition contains $20 n-20$ edges $s t$, where $\operatorname{deg}(s)=4$ and $d(t)=9$. The third edge partition contains 8 edges st, where $\operatorname{deg}(s)=6$ and $d(t)=6$. The fourth edge partition contains 8 number of edges st, where $\operatorname{deg}(s)=6$ and $d(t)=9$. The fifth edge partition contains $8 n-12$ number of edges st, where $\operatorname{deg}(s)=d(t)=9$. The Table 1 shows the edge partition of $F C C(n)$ with $n \geq 2$.

Table 1. Degree based partition of edges of $F C C(n)$.

\begin{tabular}{lll}
\hline$(d(s), d(t))$ & Frequency & Edge sets \\
\hline$(4,6)$ & 24 & $E_{1}$ \\
$(4,9)$ & $20 n-20$ & $E_{2}$ \\
$(6,6)$ & 8 & $E_{3}$ \\
$(6,9)$ & 8 & $E_{4}$ \\
$(9,9)$ & $8 n-12$ & $E_{5}$ \\
\hline
\end{tabular}


Theorem 2.1. Consider the Face-centred cubic lattice $F C C(n)$, then its general Randic index is equal to

$$
R_{\alpha}(F C C(n))= \begin{cases}1368 n-396, & \text { if } \alpha=1, \\ \frac{53 n+54}{81}, & \text { if } \alpha=-1, \\ 192 n-230+96 \sqrt{6}, & \text { if } \alpha=\frac{1}{2}, \\ \frac{20}{3 \sqrt{6}}+\frac{38 n-30}{9} & \text { if } \alpha=\frac{-1}{2}\end{cases}
$$

Proof. Let $G$ be the graph of Face-centred cubic lattice $F C C(n)$. The above results for Randić index can be proved by using Table 1 and Equation 1.2 in the following computation.

For $\alpha=1$,

$$
\begin{aligned}
R_{1}(G)= & \sum_{s t \in E(G)}(d(s) \times d(t)) \\
R_{1}(G)= & \sum_{s t \in E_{1}(G)}(d(s) \times d(t))+\sum_{s t \in E_{2}(G)}(d(s) \times d(t))+\sum_{s t \in E_{3}(G)}(d(s) \times d(t)) \\
& +\sum_{s t \in E_{4}(G)}(d(s) \times d(t))+\sum_{s t \in E_{5}(G)}(d(s) \times d(t)) \\
R_{1}(G)= & (24)(4 \times 6)+(20 n-20)(4 \times 9)+(8)(6 \times 6)+(8)(6 \times 9)+(8 n-12)(9 \times 9) \\
R_{1}(G)= & 1368 n-396 .
\end{aligned}
$$

For $\alpha=-1$, the formula of Randić index takes the following form.

$$
\begin{aligned}
R_{-1}(G)= & \sum_{s t \in E(G)} \frac{1}{(d(s) \times d(t))} \\
R_{-1}(G)= & \sum_{s t \in E_{1}(G)} \frac{1}{(d(s) \times d(t))}+\sum_{s t \in E_{2}(G)} \frac{1}{(d(s) \times d(t))}+\sum_{s t \in E_{3}(G)} \frac{1}{(d(s) \times d(t))} \\
& +\sum_{s t \in E_{4}(G)} \frac{1}{(d(s) \times d(t))}+\sum_{s t \in E_{5}(G)} \frac{1}{(d(s) \times d(t))} \\
R_{-1}(G)= & (24)\left(\frac{1}{4 \times 6}\right)+(20 n-20)\left(\frac{1}{4 \times 9}\right)+(8)\left(\frac{1}{6 \times 6}\right)+(8)\left(\frac{1}{6 \times 9}\right) \\
& +(8 n-12)\left(\frac{1}{9 \times 9}\right) \\
R_{-1}(G)= & \frac{53 n+54}{81} .
\end{aligned}
$$

For $\alpha=\frac{1}{2}$,

$$
\begin{aligned}
R_{\frac{1}{2}}(G)= & \sum_{s t \in E(G)} \sqrt{(d(s) \times d(t))} \\
R_{\frac{1}{2}}(G)= & \sum_{s t \in E_{1}(G)} \sqrt{(d(s) \times d(t))}+\sum_{s t \in E_{2}(G)} \sqrt{(d(s) \times d(t))}+\sum_{s t \in E_{3}(G)} \sqrt{(d(s) \times d(t))} \\
& +\sum_{s t \in E_{4}(G)} \sqrt{(d(s) \times d(t))}+\sum_{s t \in E_{5}(G)} \sqrt{(d(s) \times d(t))} \\
R_{\frac{1}{2}}(G)= & (24)(\sqrt{4 \times 6})+(20 n-20)(\sqrt{4 \times 9})+(8)(\sqrt{6 \times 6})+(8)(\sqrt{6 \times 9}) \\
& +(8 n-12)(\sqrt{9 \times 9}) \\
R_{\frac{1}{2}}(G)= & 192 n-230+96 \sqrt{6} .
\end{aligned}
$$


For $\alpha=-\frac{1}{2}$,

$$
\begin{aligned}
R_{-\frac{1}{2}}(G)= & \sum_{s t \in E(G)} \frac{1}{\sqrt{(d(s) \times d(t))}} \\
R_{-\frac{1}{2}}(G)= & \sum_{s t \in E_{1}(G)} \frac{1}{\sqrt{(d(s) \times d(t))}}+\sum_{s t \in E_{2}(G)} \frac{1}{\sqrt{(d(s) \times d(t))}}+\sum_{s t \in E_{3}(G)} \frac{1}{\sqrt{(d(s) \times d(t))}} \\
& +\sum_{s t \in E_{4}(G)} \frac{1}{\sqrt{(d(s) \times d(t))}}+\sum_{s t \in E_{5}(G)} \frac{1}{\sqrt{(d(s) \times d(t))}} \\
R_{-\frac{1}{2}}(G)= & (8)\left(\frac{1}{\sqrt{4 \times 6}}\right)+(20 n-20)\left(\frac{1}{\sqrt{4 \times 9}}\right)+(8)\left(\frac{1}{\sqrt{6 \times 6}}\right)+(8)\left(\frac{1}{\sqrt{6 \times 9}}\right) \\
& +(8 n-12)\left(\frac{1}{\sqrt{9 \times 9}}\right) \\
R_{-\frac{1}{2}}(G)= & \frac{20}{3 \sqrt{6}}+\frac{38 n-30}{9} .
\end{aligned}
$$

The graphical comparison of general Randić indices is depicted in Figure 3.

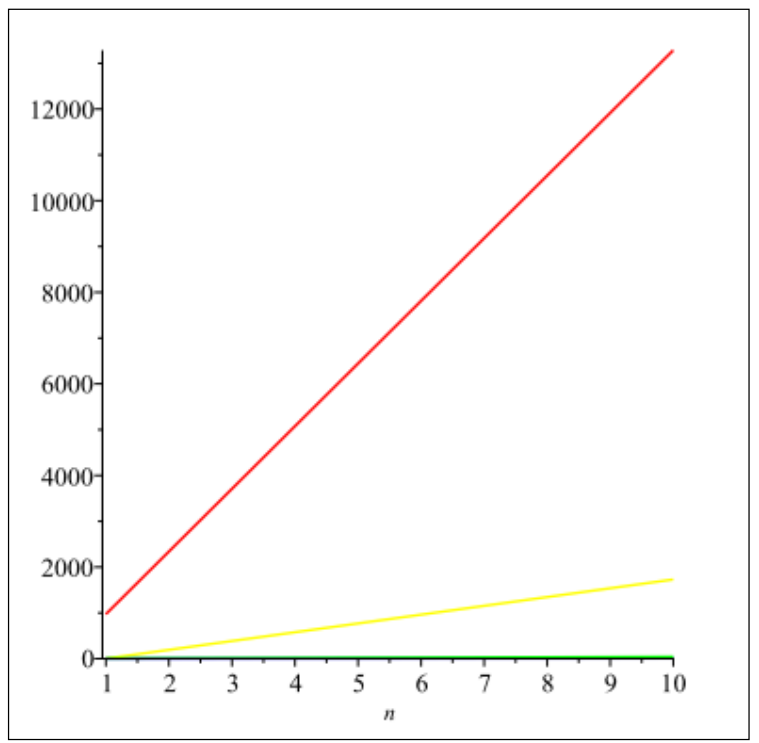

Figure 3. Comparison of general Randić indices, for $\alpha \in\{1,-1,1 / 2,-1 / 2\}$, in $2 D$ structure of Face-centred cubic lattice $F C C(n)$. The colors red, blue, yellow and green represents $R_{1}(G), R_{-1}(G) R_{\frac{1}{2}}(G), R_{-\frac{1}{2}}(G)$, respectively. We can see that in the given domain $R_{1}(G)$ is more dominating and all the indices behave differently.

Theorem 2.2. Consider the Face-centred cubic lattice $F C C(n)$, then its atomic bond connectivity $A B C$ is equal to

$$
A B C(G)=24+\frac{(10 n-10) \sqrt{11}}{3}+\frac{4 \sqrt{10}}{3}+\frac{8 \sqrt{13}}{3 \sqrt{6}}+\frac{32 n-48}{9} .
$$

Proof. Let $G$ be a the grpah of Face-centred cubic lattice $F C C(n)$. Then from the edge partition of $F C C(n)$ based on degrees of end vertices of each edge with their frequencies 
which is given in Table 1, the ABC index can be calculated by using Table 1 and Equation 1.3 in the following equation.

$$
\begin{aligned}
A B C(G)= & \sum_{s t \in E(G)} \sqrt{\frac{d(s)+d(t)-2}{d(s) \times d(t)}} \\
A B C(G)= & \sum_{s t \in E_{1}(G)} \sqrt{\frac{d(s)+d(t)-2}{d(s) \times d(t)}}+\sum_{s t \in E_{2}(G)} \sqrt{\frac{d(s)+d(t)-2}{d(s) \times d(t)}} \\
& +\sum_{s t \in E_{3}(G)} \sqrt{\frac{d(s)+d(t)-2}{d(s) \times d(t)}}+\sum_{s t \in E_{4}(G)} \sqrt{\frac{d(s)+d(t)-2}{d(s) \times d(t)}} \\
& +\sum_{s t \in E_{5}(G)} \sqrt{\frac{d(s)+d(t)-2}{d(s) \times d(t)}} \\
A B C(G)= & (24) \sqrt{\frac{4+6-2}{4 \times 6}+(20 n-20)} \sqrt{\frac{4+9-2}{4 \times 9}}+(8) \sqrt{\frac{6+6-2}{6 \times 6}} \\
& +(8) \sqrt{\frac{6+9-2}{6 \times 9}+(8 n-12)}
\end{aligned}
$$

After some easy calculations, we get:

$$
A B C(G)=24+\frac{(10 n-10) \sqrt{11}}{3}+\frac{4 \sqrt{10}}{3}+\frac{8 \sqrt{13}}{3 \sqrt{6}}+\frac{32 n-48}{9} .
$$

Theorem 2.3. Consider the Face-centred cubic lattice $F C C(n)$, then its $G A$ index is equal to

$$
G A(G)=\frac{48 \sqrt{6}+32}{5}+\frac{344 n-162}{3} .
$$

Proof. Let $G$ be the graph of Face-centred cubic lattice $F C C(n)$. The above result can be proved by using Table 1 and Equation 1.4 in the following computation. The $G A$ index is computed as below:

$$
\begin{aligned}
G A(G)= & \sum_{s t \in E(G)} \frac{2 \sqrt{d(s) d(t)}}{d(s)+d(t)} \\
G A(G)= & \sum_{s t \in E_{1}(G)} \frac{2 \sqrt{d(s) d(t)}}{d(s)+d(t)}+\sum_{s t \in E_{2}(G)} \frac{2 \sqrt{d(s) d(t)}}{d(s)+d(t)}+\sum_{s t \in E_{3}(G)} \frac{2 \sqrt{d(s) d(t)}}{d(s)+d(t)} \\
& +\sum_{s t \in E_{4}(G)} \frac{2 \sqrt{d(s) d(t)}}{d(s)+d(t)}+\sum_{s t \in E_{5}(G)} \frac{2 \sqrt{d(s) d(t)}}{d(s)+d(t)} \\
G A(G)= & (24) \frac{2 \sqrt{4 \times 6}}{4+6}+(20 n-20) \frac{2 \sqrt{4 \times 9}}{4+9}+(8) \frac{2 \sqrt{6 \times 6}}{6+6}+(8) \frac{2 \sqrt{6 \times 9}}{6+9} \\
& +(8 n-12) \frac{2 \sqrt{9 \times 9}}{9+9} \\
G A(G)= & \frac{48 \sqrt{6}+32}{5}+\frac{344 n-162}{3} .
\end{aligned}
$$




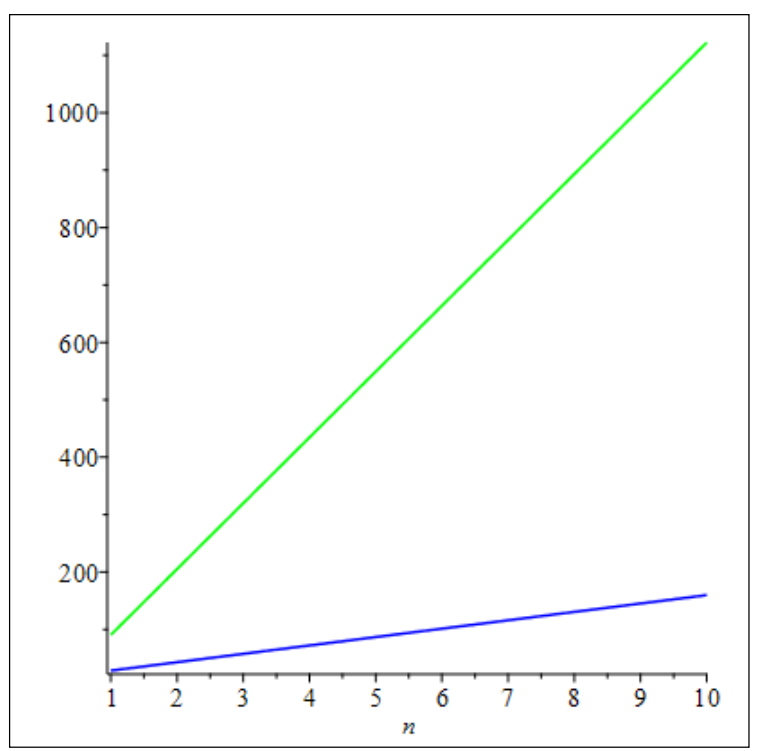

Figure 4. Comparison of atomic bond connectivity $A B C$ and geometric arithmetic index $G A$ in $2 D$ structure of Face-centred cubic lattice $F C C(n)$. The colors blue and green represents $A B C$ and $G A$ respectively. We can see that in the given domain geometric arithmetic index $G A$ is more dominating and both indices behave differently.

The graphical comparison of atomic bond connectivity $A B C$ and geometric arithmetic index $G A$ is depicted in Figure 4.

Theorem 2.4. Consider the Face-centred cubic lattice $F C C(n)$, then its first and second Zagreb index are equal to:

$$
\begin{aligned}
& M_{1}(G)=476 n+52 . \\
& M_{2}(G)=1692 n-72 .
\end{aligned}
$$

Proof. Let $G$ be the graph of $F C C(n)$. The above result can be proved by using Table 1 and Equation 1.5, Equation 1.6 in the following computation. The first Zagreb index is computed as below:

$$
\begin{aligned}
M_{1}(G)= & \sum_{s t \in E(G)}(d(s)+d(t)) \\
M_{1}(G)= & \sum_{s t \in E_{1}(G)}(d(s)+d(t))+\sum_{s t \in E_{2}(G)}(d(s)+d(t))+\sum_{s t \in E_{3}(G)}(d(s)+d(t)) \\
& +\sum_{s t \in E_{4}(G)}(d(s)+d(t))+\sum_{s t \in E_{5}(G)}(d(s)+d(t)) \\
M_{1}(G)= & (24)(4+6)+(20 n-20)(4+9)+(8)(6+6)+(8)(6+9)+(12 n-8)(9+9) \\
M_{1}(G)= & 476 n+52 .
\end{aligned}
$$

The second Zagreb index is computed below: 


$$
\begin{aligned}
M_{2}(G)= & \sum_{s t \in E(G)}(d(s) \times d(t)) \\
M_{2}(G)= & \sum_{s t \in E_{1}(G)}(d(s) \times d(t))+\sum_{s t \in E_{2}(G)}(d(s) \times d(t))+\sum_{s t \in E_{3}(G)}(d(s) \times d(t)) \\
& +\sum_{s t \in E_{4}(G)}(d(s) \times d(t))+\sum_{s t \in E_{5}(G)}(d(s) \times d(t)) \\
M_{2}(G)= & (24)(4 \times 6)+(20 n-20)(4 \times 9)+(8)(6 \times 6)+(8)(6 \times 9)+(12 n-8)(9 \times 9) \\
M_{2}(G)= & 1692 n-72 .
\end{aligned}
$$

The graphical comparison of first Zagreb index and second Zagreb index is depicted in Figure 5.

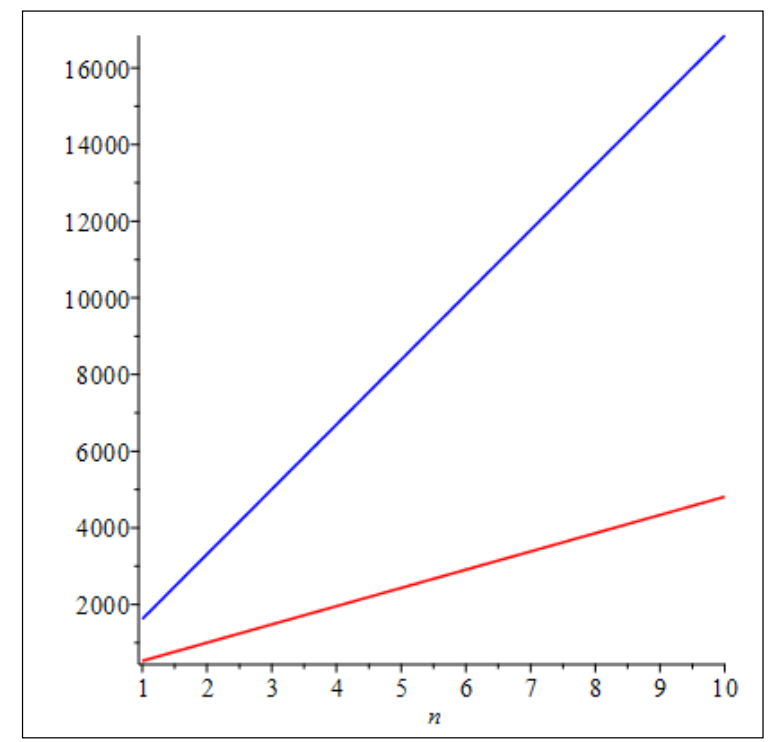

Figure 5. Comparison of first Zagreb index and second Zagreb index in $2 D$ structure of Face-centred cubic lattice $F C C(n)$. The colors red and blue represents $M_{1}(G)$ and $M_{2}(G)$ respectively. We can see that in the given domain $M_{1}(G)$ is more dominating and both indices behave differently.

Theorem 2.5. Consider the graph of $F C C(n)$, then its first and second Zagreb coindices are equal to,

$$
\begin{aligned}
& \overline{M_{1}}(G)=504 n^{2}-108 n+12 . \\
& \overline{M_{2}}(G)=1568 n^{2}-1034 n+174 .
\end{aligned}
$$

Proof. Let $G$ be the graph of $F C C(n)$. Then by using Equation (1.9) the first Zagreb coindex is computed as below:

$$
\begin{aligned}
\overline{M_{1}}(G) & =\sum_{s t \notin E(G)}(d(s)+d(t) \\
\overline{M_{1}}(G) & =2|E(G)|(|V(G)|-1)-M_{1}(G) \\
& =2(28 n+8)(9 n+5-1)-(476 n+52) \\
& =504 n^{2}-108 n+12 .
\end{aligned}
$$


Now, by using Equation (1.10) the second Zagreb coindex is computed as below:

$$
\begin{aligned}
\overline{M_{2}}(G) & =\sum_{s t \notin E(G)}(d(s) \times d(t)) \\
& =2|E(G)|^{2}-\frac{1}{2} M_{1}(G)-M_{2}(G) \\
\overline{M_{2}}(G) & =2(28 n+8)^{2}-\frac{(476 n+52)}{2}-(1692 n-72) \\
& =1568 n^{2}-1034 n+174 .
\end{aligned}
$$

The graphical comparison of first Zagreb coindex and second Zagreb coindex is depicted in Figure 6.

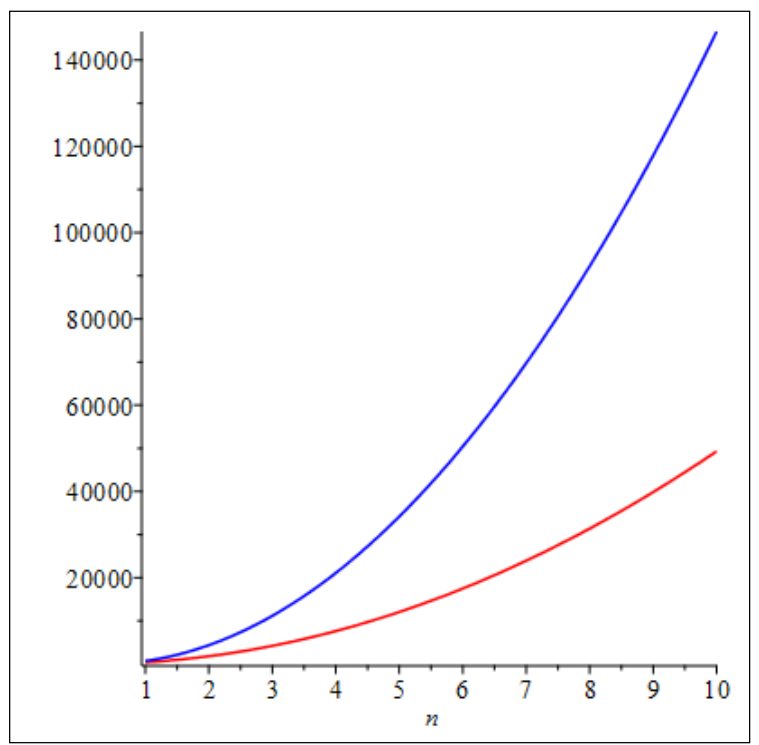

Figure 6. Comparison of first Zagreb coindex and second Zagreb coindex in $2 D$ structure of Face-centred cubic lattice $F C C(n)$. The colors red and blue represents $\overline{M_{1}}(G)$ and $\overline{M_{2}}(G)$ respectively. We can see that in the given domain $\overline{M_{2}}(G)$ is more dominating and both indices behave differently.

The Table 2 shows partition of edges of the graph Face-centred cubic lattice $F C C(n)$ depending on the sum of degrees of the neighbouring vertices of end vertices of each edge. The next Theorem shows the exact value of Sanskruti index of $F C C(n)$.

Theorem 2.6. Consider the graph $G \cong F C C(n)$, then its Sanskruti index $F C C(n)$ is equal to

$$
\begin{aligned}
S(G)= & (8)\left(\frac{33 \times 33}{33+33-2}\right)^{3}+(8)\left(\frac{24 \times 33}{24+33-2}\right)^{3}+(16)\left(\frac{30 \times 33}{30+33-2}\right)^{3} \\
& +(24)\left(\frac{36 \times 53}{36+53-2}\right)^{3}+(16)\left(\frac{30 \times 53}{30+53-2}\right)^{3}+(8)\left(\frac{33 \times 53}{33+53-2}\right)^{3} \\
& +(8)\left(\frac{53 \times 53}{53+53-2}\right)^{3}+(8)\left(\frac{53 \times 56}{53+56-2}\right)^{3}+(8 n-28)\left(\frac{56 \times 56}{56+56-2}\right)^{3} \\
& +(20 n-60)\left(\frac{36 \times 56}{36+56-2}\right)^{3} .
\end{aligned}
$$


Table 2. Degree based partition of edges of $F C C(n)$, of end vertices of each edge for $n \geq 2$.

\begin{tabular}{lll}
\hline$\left(S_{u}, S_{v}\right)$ & Number of Edges & Edge Sets \\
\hline$(33,33)$ & 8 & $E_{1}(G)$ \\
$(24,33)$ & 8 & $E_{2}(G)$ \\
$(30,33)$ & 16 & $E_{3}(G)$ \\
$(36,53)$ & 24 & $E_{4}(G)$ \\
$(30,53)$ & 16 & $E_{5}(G)$ \\
$(33,53)$ & 8 & $E_{6}(G)$ \\
$(53,53)$ & 8 & $E_{7}(G)$ \\
$(53,56)$ & 8 & $E_{8}(G)$ \\
$(56,56)$ & $8 \mathrm{n}-28$ & $E_{9}(G)$ \\
$(36,56)$ & $20 \mathrm{n}-60$ & $E_{10}(G)$ \\
\hline
\end{tabular}

Proof. Let $G$ be the graph structure of $F C C(n)$. Then by using Table 2 and equation (1.11) the Sanskruti index $S(G)$ is computed as follows.

$$
\begin{aligned}
S(G)= & \sum_{s t \in E(G)}\left(\frac{S_{s} \times S_{t}}{S_{s}+S_{t}-2}\right)^{3} \\
S(G)= & \sum_{s t \in E_{1}(G)}\left(\frac{S_{s} \times S_{t}}{S_{s}+S_{t}-2}\right)^{3}+\sum_{s t \in E_{2}(G)}\left(\frac{S_{s} \times S_{t}}{S_{s}+S_{t}-2}\right)^{3}+\sum_{s t \in E_{3}(G)}\left(\frac{S_{s} \times S_{t}}{S_{s}+S_{t}-2}\right)^{3} \\
& +\sum_{s t \in E_{4}(G)}\left(\frac{S_{s} \times S_{t}}{S_{s}+S_{t}-2}\right)^{3}+\sum_{s t \in E_{5}(G)}\left(\frac{S_{s} \times S_{t}}{S_{s}+S_{t}-2}\right)^{3}+\sum_{s t \in E_{6}(G)}\left(\frac{S_{s} \times S_{t}}{S_{s}+S_{t}-2}\right)^{3} \\
& +\sum_{s t \in E_{7}(G)}\left(\frac{S_{s} \times S_{t}}{S_{s}+S_{t}-2}\right)^{3}+\sum_{s t \in E_{8}(G)}\left(\frac{S_{s} \times S_{t}}{S_{s}+S_{t}-2}\right)^{3}+\sum_{s t \in E_{9}(G)}\left(\frac{S_{s} \times S_{t}}{S_{s}+S_{t}-2}\right)^{3} \\
& +\sum_{s t \in E_{10}(G)}\left(\frac{S_{s} \times S_{t}}{S_{s}+S_{t}-2}\right)^{3} \\
S(G)= & (8)\left(\frac{33 \times 33}{33+33-2}\right)^{3}+(8)\left(\frac{24 \times 33}{24+33-2}\right)^{3}+(16)\left(\frac{30 \times 33}{30+33-2}\right)^{3} \\
& +(24)\left(\frac{36 \times 53}{36+53-2}\right)^{3}+(16)\left(\frac{30 \times 53}{30+53-2}\right)^{3}+(8)\left(\frac{33 \times 53}{33+53-2}\right)^{3} \\
& +(8)\left(\frac{53 \times 53}{53+53-2}\right)^{3}+(8)\left(\frac{53 \times 56}{53+56-2}\right)^{3}+(8 n-28)\left(\frac{56 \times 56}{56+56-2}\right)^{3} \\
& +(20 n-60)\left(\frac{36 \times 56}{36+56-2}\right)^{3} \cdot
\end{aligned}
$$




\section{Conclusion}

In this paper, we study a well reputed lattice namely, Face-Centred Cubic Lattice $F C C(n)$ and we determined the topological indices namely, Randic index, atomic bond connectivity index, Zagreb types indices, Sanskruti index for Face-Centred Cubic Lattice $F C C(n)$. Also the topological properties of Face-Centred Cubic Lattice $F C C(n)$ are analyzed by the comparison of these topological indices using $2 \mathrm{D}$ plotting.

Acknowledgment. This research is supported by the Start-up Research Grant 2016 of United Arab Emirates University (UAEU), Al Ain, United Arab Emirates via Grant No. G00002233 and UPAR Grant of UAEU via Grant No. G00002590.

\section{References}

[1] D. Amic, D. Beslo, B. Lucic, S. Nikolic, N. Trinajstic, The vertex-connectivity index revisited, J. Chem. Inf. Comput. Sci. 38, 819-822, 1998.

[2] M. Bača, J. Horváthová, M. Mokrišová, A. Suhányiová, On topological indices of fullerenes, Appl. Math. Comput. 251, 154-161, 2015.

[3] R.J. Bie, M. K. Siddiqui, R. Razavi, M. Taherkhani, M. Najaf, Possibility of C38 and Si19Ge19 Nanocages in Anode of Metal Ion Batteries: Computational Examination, Acta Chim. Slov. 65, 303-311, 2018.

[4] B. Bollobás, P. Erdos, Graphs of extremal weights, Ars. Combi. 50, 225-233, 1998.

[5] C.R.A. Catlow, Modelling and predicting crystal structures, Interdisciplinary. Sci. Reviews. 40 294-307, 2015.

[6] G. Caporossi, I. Gutman, P. Hansen, L. Pavlovic, Graphs with maximum connectivity index, Comput. Bio. Chem. 27, 85-90, 2003.

[7] J.H. Conway, N.J.A. Sloane, Sphere packings, lattices, and groups, $2^{\text {nd }}$ ed, SpringerVerlag, New York, 1993.

[8] A.A. Dobrynin, R. Entringer, I. Gutman, Wiener index of trees: Theory and applications, Acta. Appl. Math. 66, 211-249, 2001.

[9] T. Doslic, Vertex-weighted Wiener polynomials for composite graphs, Ars. Math.Contemp. 1, 66-80, 2008.

[10] E. Estrada, L. Torres, L. Rodríguez, I. Gutman, An atom-bond connectivity index: Modelling the enthalpy of formation of alkanes, Indian. J. Chem. 37A, 849-855, 1998.

[11] W. Gao and M. K. Siddiqui, Molecular Descriptors of Nanotube, Oxide, Silicate, and Triangulene Networks, J. of. Chem., 2017, 1-10, 2017.

[12] W. Gao, M. K. Siddiqui, M. Imran, M. K. Jamil, M.R. Farahani, Forgotten Topological Index of Chemical Structure in Drugs, Saudi Pharm. J. 24, 258-267, 2016.

[13] W. Gharibi, A. Ahmad, M. K. Siddiqui, On Zagreb Indices, Zagreb Polynomials of Nanocone and Nanotubes, J. Comput. Theor. Nanosci. 13, 5086-5092, 2016.

[14] I. Gutman, C. N. Das, The first Zagreb index 30 years after, MATCH. Commun. Math. Comput. Chem. 50, 83-92, 2004.

[15] I. Gutman, K. C. Das, Some Properties of the Second Zagreb Index, MATCH. Commun. Math. Comput. Chem. 50, 103-112, 2004.

[16] I. Gutman, B. Furtula , Z. K. Vukicevic, G. Popivoda, On Zagreb indices and Coindices, MATCH. Commun. Math. Comput. Chemmm. 74, 5-16, 2015.

[17] I. Gutman, N. Trinajst, Graph theory and molecular orbitals, Total $\pi$-electron energy of alternant hydrocarbons, Chem. Phys. Lett. 17, 535-538, 1972.

[18] S.M. Hosamani, Computing Sanskruti index of certain nanostructures, J. Appl. Math. Comput. 54, 425-433, 2016.

[19] M. Imran, M.K. Siddiqui, M. Naeem, and M.A. Iqbal, On Topological Properties of Symmetric Chemical Structures, Symmetry 10, 1-20, 2018. 
[20] J.B. Liu, M.K. Siddiqui, M.A. Zahid, M. Naeem, and A.Q. Baig, Topological Properties of Crystallographic Structure of Molecules, Symmetry 10, 1-18, 2018.

[21] X. Li, I. Gutman, Mathematical aspects of Randic type molecular structure descriptors, Mathematical chemistry monographs No. 1, Kragujevac, 2006.

[22] H. Mujahed, B. Nagy, Exact formula for computing the hyper-Wiener index on rows of unit cells of the face-centred cubic lattice, An. S.t. Univ. Ovidius Constant. 26 (1), 169-187, 2018.

[23] M. Randic, On characterization of molecular branching, J. Amer. Chem. Soc. 97 (23), 6609-6615, 1975.

[24] Z. Shao, M. K. Siddiqui, and M. H. Muhammad, Computing Zagreb indices and Zagreb polynomials for symmetrical nanotubes, Symmetry. 10(7), 1-16, 2018.

[25] M.K. Siddiqui , W. Gharibi, On Zagreb indices, Zagreb polynomials of mesh derived networks, J. Comput. Theor. Nanosci. 13, 8683-8688, 2016.

[26] M.K. Siddiqui, M. Imran, and A. Ali, On Zagreb indices, Zagreb polynomials of some nanostar dendrimers, Appl. Math. Comput. 280, 132-139, 2016.

[27] M.K. Siddiqui, M. Naeem, N.A. Rahman, and M. Imran, Computing topological indices of certain networks, J. Opto. Adva. Mate. 18 (9-10), 884-892, 2016.

[28] D. Vukicevic, B. Furtula, Topological index based on the ratios of geometrical and arithmetical means of end-vertex degrees of edges, J. Math. Chem. 46, 1369-1376, 2009.

[29] C. Wang, J. Liu, and S. Wang, Sharp upper bounds for multiplicative Zagreb indices of bipartite graphs with given diameter, Disc. Appl. Math. 227, 156-165, 2017.

[30] S. Wang, C. Wang, and J. Liu, On extremal multiplicative Zagreb indices of trees with given domination number, Appl. Math. Comput. 332, 338-350, 2018.

[31] H. Wiener, Structural determination of paraffin boiling points, J. Am. Chem. Soc. 69, 17-20, 1947. 